Communications in Physics, Vol. 24, No. 3 (2014), pp. 201-206

DOI:10.15625/0868-3166/24/3/3817

\title{
MASS OF THE LIGHTEST NEUTRAL CP-EVEN HIGGS IN THE SUPERSYMMETRIC MINIMAL REDUCED 3-3-1 MODEL
}

\author{
HA THANH HUNG \\ Department of Physics, Hanoi University of Education No. 2 \\ LE THO HUE \\ Institute of Physics, Vietnam Academy of Science and Technology \\ NGUYEN THI THU HANG \\ Department No. 5, The University of Fire Fighting \& Prevention \\ PHAM NGOC THU \\ Department of Mathematics-Physics-Computer Science, Tay Bac University \\ E-mail: hthung80@gmail.com \\ Received 31 March 2014 \\ Accepted for publication 15 May 2014
}

\begin{abstract}
We calculate the mass of the lightest neutral CP-even Higgs in the frame work of the supersymmetric reduced minimal 3-3-1 model (SUSYRM331) at one-loop level. Like MSSM, at tree level this value of mass is smaller than that of $m_{Z}=92 \mathrm{GeV}$, inconsistent with value of $125 \mathrm{GeV}$ measured by recent experiment. In this work we prove that the mass of the lightest neutral CP-even Higgs will get the consistent value if loop corrections are included.
\end{abstract}

Keywords: standard model and byond.

\section{INTRODUCTION}

The detection of the neutral CP-even Higgs with mass around $125 \mathrm{GeV}$ by experiment at LHC $[1,2]$ has again confirmed the success of the Standard Model (SM). Beside that many other well-known results of experiments suggest the necessary to study new models beyond the SM, for example the non-zero mass and the oscillation of neutrinos, the existence of dark matter in the universe, the hierachy in mass spectrum of the SM, the question of the existence of three fermion families, .... Among the well-known models beyond the SM, the supersymmetric minimal reduced 33-1 model (SUSYRM331) was recently built with many interesting properties. It is the supersymmetric version of the reduced minimal 3-3-1 model (RM331) [3] which contains the simplest Higgs content comparing with well-known 3-3-1 models. It is noted that the SUSYRM331 not only keeps many interesting property of the RM331 model but also solves the dark matter problem unsolved by normal 3-3-1 models. In the Higgs spectrum of the SUSYRM331, it was shown in $[5,6]$ that at the tree level the lightest neutral CP-even Higgs has a mass smaller than mass of the $Z$ boson. The authors in [6] indicated a analytic formula of this mass as $m_{H^{0}}^{2}=m_{Z}^{2} \cos ^{2} 2 \gamma+\mathscr{O}(\varepsilon) \times m_{W}^{2}$, 
where $\gamma$ is defined as the ratio of the vacuum expection values of two neutral Higgs $\rho^{0}$ and $\rho^{0 \prime}$, i.e $\tan \gamma=v / v^{\prime}$. The quantity $\varepsilon=m_{W}^{2} / m_{V}^{2} \ll 1$ so $m_{H^{0}} \simeq m_{Z}|\cos 2 \gamma|<m_{Z}=92 \mathrm{GeV}$. This value is inconsistent with recent experiments which have detected only one Higgs with mass of $125 \mathrm{GeV}$. So it is necessary to calculate this lightest Higgs mass including loop-corrections and consider whether the full mass can reach the experimental value. At the tree level, Ref. [3] has shown the numerical result being consistent with that shown in [6]. In addition they also investigated numerically the lightest mass including one-loop correction of the top and stop quark and showed that the mass values can reach the value of $125 \mathrm{GeV}$. It is needed to recall that because top quark is the heaviest quark in the Minimal Supersymmetric Standard Model (MSSM), the correction relating with top and stop quark in this model is the largest contribution to the mass of the lightest neutral CP-even Higgs. The difference may happen in case of the SUSYRM331 because it contains many other heavy quarks and their superpartners, namely the exotic quarks. This problem was not considered in the [3]. Hence, our work will investigate more details one-loop contributions of top quark, exotic quarks and their superpartners to the mass of the lightest neutral CP-even Higgs in the SUSYRM331. In particularly, we will use the method given from [8] to estimate the mass of the lightest neutral CP-even Higgs at the tree as well as one-loop level including only heavy quarks and their superpartners. The mass of this Higgs at two levels will be presented in sections II and III, respectively. The summarization of our work is in the section IV.

\section{MASS OF NETRAL HIGGS AT TREE LEVEL}

The Higgs sector in this model is given as follows [4,5], in particularly

$$
\rho=\left(\begin{array}{c}
\rho^{+} \\
\rho^{0} \\
\rho^{++}
\end{array}\right) \sim(\mathbf{1}, \mathbf{3},+1), \quad \hat{\chi}=\left(\begin{array}{c}
\chi^{-} \\
\chi^{--} \\
\chi^{0}
\end{array}\right) \sim(\mathbf{1}, \mathbf{3},-1),
$$

and

$$
\rho^{\prime}=\left(\begin{array}{c}
\rho^{\prime-} \\
\rho^{\prime 0} \\
\rho^{\prime--}
\end{array}\right) \sim\left(\mathbf{1}, \mathbf{3}^{*},-1\right), \quad \chi^{\prime}=\left(\begin{array}{c}
\chi^{\prime+} \\
\chi^{\prime++} \\
\chi^{\prime 0}
\end{array}\right) \sim\left(\mathbf{1}, \mathbf{3}^{*},+1\right) .
$$

All neutral Higgs components having non-zero vacuum expectations (VEV) are defined as $\rho^{0}=$ $\frac{v+H_{\rho}+i F_{\rho}}{\sqrt{2}}, \rho^{\prime 0}=\frac{v^{\prime}+H_{\rho^{\prime}}+i F_{\rho^{\prime}}}{\sqrt{2}}, \chi=\frac{w+H_{\chi}+F_{\chi}}{\sqrt{2}}$ and $\chi^{\prime}=\frac{w^{\prime}+H_{\chi^{\prime}}+F_{\chi^{\prime}}}{\sqrt{2}}$, where $v, v^{\prime}, w$ and $w^{\prime}$ are respective VEVs of these Higgses. We will use the following notations given in [4,6]: $t_{\gamma} \equiv \tan \gamma=v / v^{\prime}$, $t_{\beta} \equiv \tan \beta=w / w^{\prime}, m_{V}^{2}=\frac{g^{2}}{4}\left(w^{2}+w^{\prime 2}\right), m_{W}^{2}=\frac{g^{2}}{4}\left(v^{2}+v^{\prime 2}\right)=80.4^{2}\left[\mathrm{GeV}^{2}\right]$. Here $m_{V}$ and $m_{W}$ are masses of the heavy $V$ and $W$ gauge bosons respectively. For the simple in estimating value of the Lightest Neutral CP-Even Higgs (LNEH), based on the limit case supposed in [8] for MSSM we consider the case of the SUSYRM331 with some parameters are fixed as follows: $\beta, \gamma \rightarrow \pi / 2$. As a consequence we have $v^{\prime}, w^{\prime} \rightarrow 0$ compared with corresponding values of $v$ and $w$. Therefore it can be supposed that $\rho^{\prime}, \chi^{\prime} \rightarrow 0$ and $m_{W}=g v / 2, m_{V}=g w / 2$. Now we can ignore all terms relating with $\chi^{\prime}$ and $\rho^{\prime}$ in the superpotential given in $[3,6]$. In addition we define some new parameters such as

$$
m_{1}^{2}=\frac{1}{4} \mu_{\chi}^{2}+m_{\chi}^{2}, \quad m_{2}^{2}=\frac{1}{4} \mu_{\rho}^{2}+m_{\rho}^{2}, \quad \frac{H_{\chi}+w}{\sqrt{2}} \rightarrow \phi_{1} \quad \text { and } \quad \frac{H_{\rho}+v}{\sqrt{2}} \rightarrow \phi_{2} .
$$


With the limit supposed above, at the tree level the superpotential relating with neutral CP-even Higgses is

$$
V_{\text {SUSYRM } 331} \rightarrow V_{0}=m_{1}^{2} \phi_{1}^{2}+m_{2}^{2} \phi_{2}^{2}+\frac{g^{2}\left(t^{2}+2\right)}{12}\left[\phi_{1}^{4}-2 k \phi_{1}^{2} \phi_{2}^{2}+\phi_{2}^{4}\right]
$$

where $t^{2} \equiv \frac{g^{\prime 2}}{g^{2}}=\frac{6 s_{W}^{2}}{1-4 s_{W}^{2}}$ and $k=\left(t^{2}+1\right) /\left(t^{2}+2\right)$. The minimal condition of the $V_{0}$ corresponds to two below equations

$$
\begin{aligned}
& \left.\frac{\partial V_{0}}{\partial \phi_{1}}\right|_{\phi_{1}=w / \sqrt{2}, \phi_{2}=v / \sqrt{2}}=0 \rightarrow m_{1}^{2}=-\frac{t^{2}+2}{3}\left(m_{V}^{2}-k m_{W}^{2}\right), \\
& \left.\frac{\partial V_{0}}{\partial \phi_{2}}\right|_{\phi_{1}=w / \sqrt{2}, \phi_{2}=v / \sqrt{2}}=0 \rightarrow m_{2}^{2}=\frac{t^{2}+2}{3}\left(k m_{V}^{2}-m_{W}^{2}\right) .
\end{aligned}
$$

Because masses of these two Higgses relate with $\frac{\partial^{2} V_{0}}{\partial \phi_{i} \phi_{j}}(i, j=1,2)$, we have the mass term of the Higgses as follows

$$
\frac{2\left(t^{2}+3\right) m_{V}^{2}}{3}\left(\begin{array}{ll}
\phi_{1}, & \phi_{2}
\end{array}\right)\left(\begin{array}{cc}
1 & -k \sqrt{\varepsilon} \\
-k \sqrt{\varepsilon} & \varepsilon
\end{array}\right)\left(\begin{array}{c}
\phi_{1} \\
\phi_{2}
\end{array}\right),
$$

where $\varepsilon=m_{W}^{2} / m_{V}^{2} \ll 1$. Squared masses of the physical neutral Higgses are eigenvalues of matrix in (6). They are determined as

$$
m_{h, H}^{2}=\frac{\left(t^{2}+2\right) m_{V}^{2}}{3}\left[1+\varepsilon \mp \sqrt{1+\varepsilon\left(4 k^{2}-2\right)+\varepsilon^{2}}\right] .
$$

Because $\varepsilon \ll 1$ so we can obtain the approximation for the lightest neutral Higgs mass as $m_{h}^{2} \simeq$ $m_{W}^{2} / c_{W}^{2}+\mathscr{O}(\varepsilon) \times m_{W}^{2}$ or $m_{h}^{2} \simeq m_{Z}^{2}=92 \mathrm{GeV}$. This result is consistent with conclusions of previous publications $[3,6]$. In the next section we will calculate mass of this Higgs including one-loop correction from heavy quarks and their superpartners.

\section{CORRECTION AT ONE LOOP LEVEL}

To calculate the loop correction of Higgs masses we need to find out the Yukawa interactions of quarks as well as the soft terms relating with their superpartners. For Yukawa terms, concentrating only on heavy quarks, we use the approach given in [3], namely the term mainly creating masses of top and exotic quarks can be supposed simply as

$$
\mathscr{L}_{\text {mass }}^{\mathrm{q}}=\lambda_{i i}^{J} Q_{i L} \chi J_{i L}^{c}+\lambda_{t}^{\prime \prime} Q_{3 L} \rho t_{L}^{c}+\frac{k_{u 1 a}}{\Lambda} \varepsilon_{m n p}\left(Q_{1 L} \rho_{m} \chi_{p}\right) u_{a}^{c},
$$

where $a=1,2,3$ and $i=2,3$ being the family indices. Noting that the lagrangian (8) contains only terms relating with Higgses $\chi$ and $\rho$ because of our above assumption that the mass of the lightest Higgs are only contributed from these Higgses. In addition, it can be proved that the last term in (8) must be much smaller than other terms in order to avoid large mixing among flavor normal quarks. Therefore this term can be ignored when we consider only heavy quarks. So only two terms generating masses for top and $J_{2,3}$ quarks are

$$
m_{J_{2,3}}^{2}=\lambda_{22,33}^{J} \phi_{1}^{2}, \quad m_{t}^{2}=\lambda_{t}^{\prime \prime 2} \phi_{2}^{2}
$$


For the squarks, apart from Yukawa interactions, their masses also come from the soft and D terms. We can ignore the D term in this case and assume that masses are given in the simplest case as follows

$$
m_{\tilde{J}_{2,3}}^{2} \equiv m_{\tilde{J}_{2,3} L}^{2}=m_{\tilde{J}_{2,3} R}^{2}=\lambda_{22,33}^{J} \phi_{1}^{2}+\tilde{m}_{J}^{2}, \quad m_{\tilde{t}}^{2}=m_{\tilde{t} L}^{2}=m_{\tilde{t} R}^{2}=\lambda_{t}^{\prime \prime 2} \phi_{2}^{2}+\tilde{m}_{t}^{2} .
$$

From this we have

$$
\frac{\partial\left(m_{t}^{2}\right)}{\partial\left(\phi_{1}\right)}=\frac{\partial\left(m_{\tilde{t}}^{2}\right)}{\partial\left(\phi_{1}\right)}=0, \quad \frac{\partial\left(m_{t}^{2}\right)}{\partial\left(\phi_{2}\right)}=\frac{\partial\left(m_{\tilde{t}}^{2}\right)}{\partial\left(\phi_{2}\right)}=2 \lambda^{\prime \prime 2} \phi_{2}
$$

and

$$
\frac{\partial\left(m_{J_{2,3}}^{2}\right)}{\partial\left(\phi_{1}\right)}=\frac{\partial\left(m_{\tilde{J}_{2,3}}^{2}\right)}{\partial\left(\phi_{1}\right)}=2\left(\lambda_{22,33}^{J}\right)^{2} \phi_{1}, \quad \frac{\partial\left(m_{J_{2,3}}^{2}\right)}{\partial\left(\phi_{2}\right)}=\frac{\partial\left(m_{\tilde{J}_{2,3}}^{2}\right)}{\partial\left(\phi_{2}\right)}=0
$$

which will be very useful when we calculate the squared Higgs mass matrix from the potential included the one-loop correction, namely

$$
V_{1}(Q)=V_{0}(Q)+\Delta V_{1}(Q),
$$

where

$$
\Delta V_{1}(Q)=\frac{1}{64 \pi^{2}} \operatorname{Str}\left[\mathscr{M}^{4}\left(\ln \frac{\mathscr{M}^{2}}{Q^{2}}-c\right)\right] .
$$

Here $Q$ is some renomalization scale and $\mathscr{M}^{2}$ is the field-dependent generalized squared mass matrix and the supertrace is defined as

$$
\operatorname{Str} f\left(\mathscr{M}^{2}\right)=\sum_{i}(-1)^{2 J_{i}}\left(2 J_{i}+1\right) f\left(m_{i}^{2}\right)
$$

with $J_{i}$ is the spin of the field having mass $m_{i}$. We consider the contribution to $\Delta V_{1}$ from only top, $J_{2,3}$ quarks and their superpartners, namely

$$
\begin{aligned}
\Delta V_{1}(Q)=\frac{3}{16 \pi^{2}} & \times\left[\sum_{i=2}^{3} 2 m_{J_{i}}^{4} \ln \left(\frac{m_{\tilde{J}_{i}}^{2}}{m_{J_{i}}^{2}}\right)+\left(m_{\tilde{J}_{i}}^{4}-m_{J_{i}}^{4}\right)\left(\ln \frac{m_{\tilde{J}_{i}}^{4}}{Q^{2}}-c\right)\right. \\
& \left.+2 m_{t}^{4} \ln \left(\frac{m_{\tilde{t}}^{2}}{m_{t}^{2}}\right)+\left(m_{\tilde{t}}^{4}-m_{t}^{4}\right)\left(\ln \frac{m_{\tilde{t}}^{4}}{Q^{2}}-c\right)\right] .
\end{aligned}
$$

The minimal condition of this potential corresponds to series of two below equations,

$$
\left.\frac{\partial V_{1}}{\partial \phi_{1}}\right|_{\phi_{1}=w / \sqrt{2}, \phi_{2}=v / \sqrt{2}}=0,\left.\quad \frac{\partial V_{1}}{\partial \phi_{2}}\right|_{\phi_{1}=w / \sqrt{2}, \phi_{2}=v / \sqrt{2}}=0,
$$

where

$$
\begin{aligned}
& \frac{\partial V_{1}}{\partial \phi_{1}}=\frac{\partial V_{0}}{\partial \phi_{1}}+\frac{\partial \Delta V_{1}}{\partial \phi_{1}} \\
& \frac{\partial V_{1}}{\partial \phi_{2}}=\frac{\partial V_{0}}{\partial \phi_{2}}+\frac{\partial \Delta V_{1}}{\partial \phi_{2}}
\end{aligned}
$$


Similarly to the case of MSSM considered in [8], we can choose the renomalization scale $Q$ so that $\frac{\partial V_{0}}{\partial \phi_{2}}=0$ and $\frac{\partial \Delta V_{1}}{\partial \phi_{2}}=0$. As the result we obtain value of $m_{2}^{2}$ the same as that given in (5) while $m_{1}^{2}$ is different. In addition from the condition $\frac{\partial \Delta V_{1}}{\partial \phi_{2}}=0$ we have

$$
\left(m_{\tilde{t}}^{2}-m_{t}^{2}\right)\left(\ln \frac{m_{\tilde{t}}^{2}}{Q}-c\right)=-\left(m_{\tilde{t}}^{2}-m_{t}^{2}\right)-2 m_{t}^{2} \ln \frac{m_{\tilde{t}}^{2}}{m_{t}^{2}}
$$

This leads to the consequence that

$$
\frac{\partial^{2} V_{1}}{\partial \phi_{2}^{2}}=\frac{\partial^{2} V_{0}}{\partial \phi_{2}^{2}}+\frac{3 g^{2}}{8 \pi^{2}} \frac{m_{t}^{4}}{m_{W}^{2}} \ln \left(\frac{m_{\tilde{t}}^{4}}{m_{t}^{4}}\right)
$$

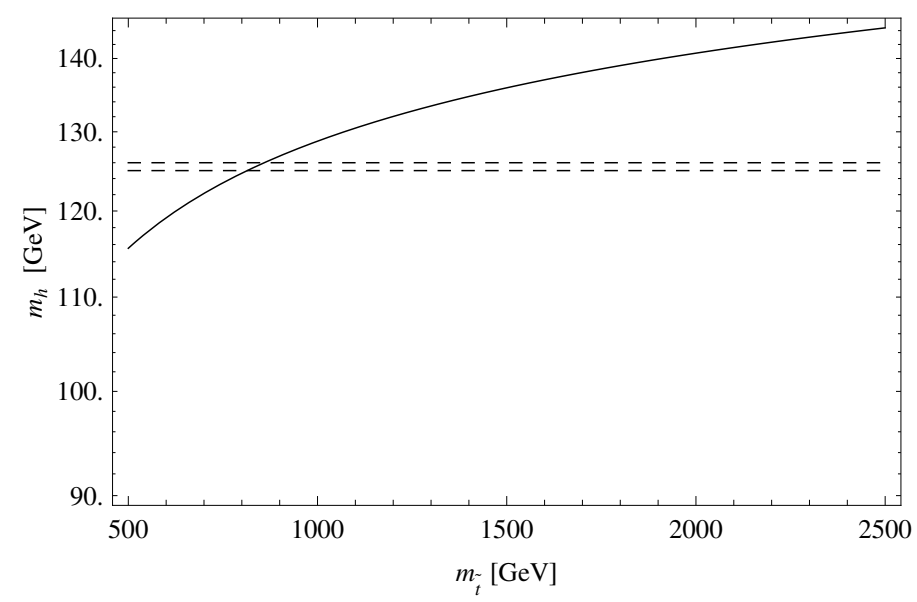

Fig. 1. Mass of the lightest CP-even neutral Higgs including one-loop correction of heavy quarks and squarks. The black curves present the mass in the framework of the SUSYRM331 as function of stop quark. Two dashed lines correspond to 125 and 126 $\mathrm{GeV}$.

We will prove that the second term in (20) is the main contribution from one-loop correction to the lightest neutral CP-even Higgs mass. Firstly, we can prove that $\frac{\partial^{2} \Delta V_{1}}{\partial \phi_{1} \partial \phi_{2}}=0$ so $\frac{\partial^{2} V_{1}}{\partial \phi_{1} \partial \phi_{2}}=\frac{\partial^{2} V_{0}}{\partial \phi_{1} \partial \phi_{2}}$. Secondly, the analytic formula of $\frac{\partial^{2} \Delta V_{1}}{\partial \phi_{1}^{2}}$ can be found by inserting $Q$ in (19) into the first equation of (18) to determine $m_{2}^{2}$ according to masses of the quarks and squarks. Value of $\frac{\partial^{2} \Delta V_{1}}{\partial \phi_{1}^{2}}$ is different from that of $\frac{\partial^{2} \Delta V_{0}}{\partial \phi_{1}^{2}}$ but based on (6) the mass term of Higgses are written as follows

$$
\frac{2\left(t^{2}+3\right) m_{V}^{2}}{3}\left(\begin{array}{ll}
\phi_{1}, & \phi_{2}
\end{array}\right)\left(\begin{array}{cc}
1+a & -k \sqrt{\varepsilon} \\
-k \sqrt{\varepsilon} & \varepsilon+b
\end{array}\right)\left(\begin{array}{c}
\phi_{1} \\
\phi_{2}
\end{array}\right)
$$


where

$$
\begin{aligned}
& \frac{2\left(t^{2}+3\right) m_{V}^{2}}{3} \times a=\left.\frac{\partial^{2} \Delta V_{1}}{\partial \phi_{1}^{2}}\right|_{\phi_{1}=w / \sqrt{2}, \phi_{2}=v / \sqrt{2}}, \\
& \frac{2\left(t^{2}+3\right) m_{V}^{2}}{3} \times b=\frac{3 g^{2}}{16 \pi^{2}} \frac{m_{t}^{4}}{m_{W}^{2}} \ln \left(\frac{m_{t}^{4}}{m_{t}^{4}}\right) .
\end{aligned}
$$

Now mass of the lightest Higgs is

$$
m_{h}^{2}=\frac{\left(t^{2}+3\right) m_{V}^{2}}{3}\left[(1+a)+(\varepsilon+b)-\sqrt{(1+a-\varepsilon-b)^{2}+4 k^{2} \varepsilon}\right] .
$$

In case of $a, b \ll 1$ we have $m_{h}^{2} \simeq m_{Z}^{2}+\frac{3 g^{2}}{16 \pi^{2}} \frac{m_{t}^{4}}{m_{W}^{2}} \ln \left(\frac{m_{t}^{4}}{m_{t}^{4}}\right)$ which is the same as shown in MSSM [8]. With $m_{Z}=92 \mathrm{GeV}, m_{t}=175 \mathrm{GeV}$ and $m_{W}=80.4 \mathrm{GeV}$, the values of $m_{h}$ as a function of $m_{\tilde{t}}$ is illustrated in the figure 1. It is easy to see that the lightest Higgs mass can reach the value of $125-126 \mathrm{GeV}$ measured by recent experiments at LHC. So the SUSYRM331 is still not excluded.

\section{CONCLUSION}

In this paper we have established formula to calculate the mass of the lightest neutral CPeven Higgs mass in the frame work of the SUSYRM331 at one-loop level. Firstly, at tree level with large values of $\tan \beta$ and $\tan \gamma$ we have proved that this mass is equal to mass of the $Z$ boson. At one-loop level where loop corrections are much small than $\mathrm{SU}(3)_{\mathrm{L}}$ scale, we obtain the formula of this lightest mass as $m_{h}^{2} \simeq m_{Z}^{2}+\frac{3 g^{2}}{16 \pi^{2}} \frac{m_{t}^{4}}{m_{W}^{2}} \ln \left(\frac{m_{t}^{4}}{m_{t}^{4}}\right)$. This result shows that the SUSYRM331 contains a lightest neutral CP-even Higgs with mass being consistent with experiments if $m_{\tilde{t}}$ is large enough. Our work only considers the largest one-loop contributions from heavy quarks and their superpartners. A more detail investigation including contribution from other quarks as well as mixing of left- and right-squarks should be investigated in order to constrain the parameter space of the model.

\section{ACKNOWLEDGMENT}

This research is funded by Vietnam National Foundation for Science and Technology Development (NAFOSTED) under grant number 103.01-2011.63.

\section{REFERENCES}

[1] The ATLAS Collaboration, Phys.Lett. B 716 (2012) 1, arXiv:1207.7214.

[2] The CMS Collaboration, G. Aad et al., Phys. Lett. B 716 (2012) 30, arXiv:1207.7235.

[3] J. G. Ferreira, Jr, P. R. D. Pinheiro, C. A. de S. Pires, P. S. Rodrigues da Silva, Phys. Rev. D 84 (2011) 095019.

[4] D. T. Huong, L. T. Hue, M. C. Rodriguez, H. N. Long, Nucl. Phys. B 870 (2013) 293.

[5] J. G. Ferreira, C. A. de S. Pires, P. S. Rodrigues da Silva, A. Sampieri, Phys. Rev. D 88 (2013) 105013.

[6] N. H. Thao, L. T. M. Phuong and L. T. Hue, Communications in Physics 23 (2013) 203, link http://vjs.ac.vn/index.php/cip/article/view/3237.

[7] J. Beringer et al. (Particle Data Group), Phys. Rev. D 86 (2012) 010001.

[8] J. Ellis, G. Ridolfi and F. Zwirner, Phys. Lett. B 257 (1991) 83. 